Artículo de reflexión

Cuestiones de Filosofía

ISSN: 0123-50-95

E-ISSN: 2389-9441

Vol. 5 - N² 24 Enero- junio, año 2019

pp. $65-86$

\title{
Enseñanza filosofante. Una perspectiva transescolar
}

\section{Philosophizing teaching: a trans-scholar perspective}

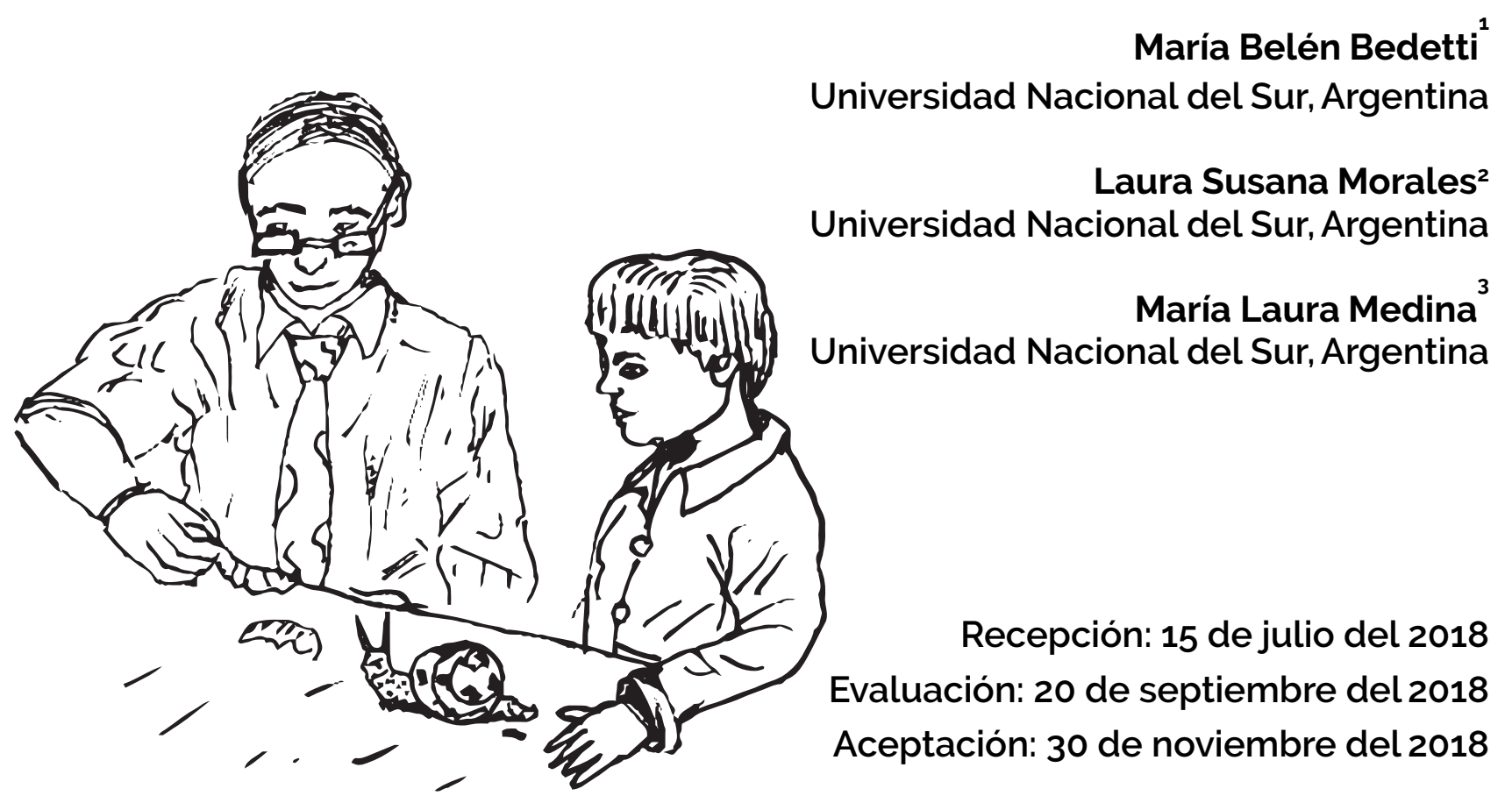

1 Profesora de Filosofía. Universidad Nacional del Sur. Bahía Blanca. Correo electrónico: belen.bedetti@uns.edu.ar

2 Profesora de Filosofía. Universidad Nacional del Sur. Bahía Blanca. Correo electrónico: Imorales@uns.edu.ar

3 Profesora de Filosofía. Universidad Nacional del Sur. Bahía Blanca. Correo electónico: laura.medina@uns.edu.ar 


\section{Resumen:}

Pensar en el objeto de enseñanza de la filosofía nos enfrenta a la siguiente dicotomía: enseñar filosofía versus enseñar a filosofar. Dicha dicotomía ha sido abordada por numerosos pensadores, entre los que se destacan Hegely Kant como representantes de cada una de las posiciones enfrentadas. Sin embargo, reflexionar sobre cada postura de manera absoluta, nos conduce a perder la riqueza que nos proporciona la experiencia. Sin negar las tensiones y conflictos en torno al debate que suscita tal dicotomía, intentamos construir una perspectiva de la enseñanza filosofante como mirada crítica de las instituciones y su vínculo con la filosofía y otros saberes. Para ello, reconocemos el florecimiento de distintas prácticas filosóficas además de las ya instaladas. Tal es el caso de la filosofía para (y con) niños, cuya particularidad es el exceder los muros de las aulas. El impacto de dichas prácticas filosofantes invita a preguntarnos si es lícito definir a todas aquellas prácticas que escapan al formato de las instituciones educativas desde la negación. De igual manera, cómo podemos nombrarlas y pensarlas con una entidad propia, sin limitarnos a recurrir a la ausencia de lo otro. Así, cobra fuerza la concepción de la filosofía desde una perspectiva transescolar, por cuanto busca presentar nuevos horizontes de análisis, sin reducirnos a pensar las prácticas de filosofía como académicas, extraacadémicas o no académicas. Empero, esta idea no está en contra de la presencia de la filosofía en el espacio público académico, ya que permite abrir una fisura para la deslegitimación de prácticas academicistas y escolarizantes.

Palabras clave: educación, filosofía, escolar, escuela, enseñanza.

\section{Abstract:}

To think in the reason of philosophy teaching face us against the following dichotomy: to teach philosophy or to teach to philosophize. That dichotomy has been approached for a great number of thinkers. Among those, we can highlight Hegel and Kant as representatives of each of the confronted positions. However, reflecting on each position in an absolute way leads us to lose the richness that the experience delivers us. Without denying the tensions and conflicts around the debate that arises from this dichotomy, we try to build a perspective about the philosophizing teaching as a critical look of institutions and their link with philosophy and other knowledge. To achieve this, we acknowledge the flourishing of different philosophical practices 
besides those already installed. Such is the case of the philosophy for (and with) children, whose particularity is to overcome the walls of the classrooms. The impact of such philosophizing practices invites us to ask ourselves if it is lawful to define all those practices that scape the template of the educative institutions by denying it. Likewise, how can we name them and think on them as an entity by itself, without bounding ourselves to appeal the absence of the other. Thus, the conception of philosophy takes strength from a trans-scholar perspective, because it tries to present new horizons of the analysis, without reducing us to think about the practices of philosophy as being either academic, extra-academic, or non-academic ones. Notwithstanding, this idea is not against the presence of philosophy in the academic and public space, because it allows us to open a breach to delegitimize academic and schooling practices.

Key-word: education, philosophy, school, school, teaching

\section{Enseignement philosophique Une perspective trans-scolaire}

\section{Résumé:}

Penser au sujet d'enseignement de la philosophie nous affronte à la suivante dichotomie: «enseigner philosophie contre enseigner à philosopher. » Celle-là a été étudiée par de diverses penseurs, parmis lesquels se trouvent Hegel et Kant, comme les représentants de chacune des pensées complètement opposées. Néanmoins, réfléchir sur chacune parmis elles d'une manière absolute nous emporte à perdre la richesse qui nous donne l'expérience. En admettant les tensions et les conflits autour du débat suscité par la dichotomie déjà exposée, nous essayons de construit une perspective de l'enseignement philosophique comme témoin critique des écoles et institutions, et son lien avec la philosophie et les autres sujets d'étude. Pour arriver à cela, nous reconnaissons l'apparition de diverses pratiques philosophiques plus des autres déjà mises en pratique. Voilà le cas de la philosophie pour (et avec) des enfants, dont, sa particularité est de dépasser les murs des salles de classe. L'impact de celles pratiques philosophiques nous invite à nous poser des questions tels que si c'est bien de définir toutes les autres pratiques qui ne sont pas inclues dans les formats des institutions éducatives à partir de la négation. Également, comment pouvons-nous les nommer et les penser avec un nom propre, sans nous limiter à recourir à l'absence de l'autre. C'est comme cela que la conception de la philosophie 
dès une perspective trans-scolaire devient plus forte, car elle cherche pouvoir présenter de nouveaux horizons à analiser, sans nous réduit à penser les pratiques de philosophie comme des académiques, extra-académiques ou non académiques. Cependant, cette idée n'est pas contre la présence de la philosophie dans l'espace publique, car cela permet d'ouvrir une opportunité pour la délégitimassions des pratiques académiques et scolaires.

Mots-clés : éducation, philosophie, école, école, enseignement.

\section{Ensino filosofante. Uma perspectiva transescolar}

\section{Resumo:}

Pensar no objeto de ensino da filosofia nos apresenta a seguinte dicotomia: ensinar filosofia versus ensinar a filosofar. Essa dicotomia tem sido abordada por numerosos pensadores, entre os que se destacam Hegel y Kant como representantes de cada uma das posições enfrentadas. Embora, refletir sobre cada postura de maneira absoluta, nos conduz a perder a riqueza que nos proporciona a experiência. Não se pode negar as tensões e conflitos sobre o debate que sucita tal dicotomia, tentamos construir uma perspectiva do ensino filosofante como olhar critico das instituições e seu vinculo com a filosofia e outros saberes. Por isso, reconhecemos o florescimento de distintas práticas filosóficas além das instaladas. Nesse caso da filosofia para le com) crianças, cuja particularidade é exceder as paredes das aulas. O impacto dessas práticas filosofantes convida a perguntar-nos se é licito definir a todo aquilo como prática que escapam do formato das instituições educacionais desde a negação. Desta maneira, como podemos nombra-las e pensa-las com uma entidade própria, sem limitantes para recorrer à ausência do outro. Assim, a concepção da filosofia tem força na perspectiva transescolar, enquanto busca apresentar horizontes novos de análises, sem reduzir-nos a pensar as práticas da filosofia como acadêmicas, extra acadêmicas ou não acadêmicas. Além, esta ideia não está contra a presencia da filosofia no espaço publico acadêmico, pois permite abrir uma fissura para a deslegitimação de práticas academistas e escolarizam-te.

Palavras chave: educação, filosofia, escolar, escola, ensino 


\section{Introducción}

Este artículo tiene por objetivo presentar una concepción de la filosofía que busca nuevos horizontes de análisis, al pensar las prácticas filosóficas en el espacio público de forma tal que permitan abrir una fisura en las prácticas tanto academicistas como escolarizantes. Asi se ha gestado el concepto de transescolaridad.

Este concepto es abordado en tres apartados, a saber: Las prácticas filosóficas institucionales alternativas desde la filosofía filosofante, La perspectiva transescolar y Aportes y rupturas de la perspectiva transescolar a la formación. En el primero, se da cuenta de nuevas prácticas filosóficas, muchas de ellas ya consolidadas, que reeditan el clásico debate entre las posturas de enseñar filosofía versus enseñar a filosofar, y cómo han reconfigurado la situación de la filosofia como disciplina. Esta cuestión, sin embargo, no es reflejada en la nominación que reciben al hacerlo desde la falta o la negación, verbigracia: "filosofía extra-académica" o "no académica".

Como fruto de este debate, y en la búsqueda de una superación que no niega tensiones, se analizan aspectos filológicos e históricos del concepto de escuela en Occidente, que permiten fundar y posicionar la perspectiva "transescolar", título del segundo apartado. Mientras que en el tercero, tal como lo indica su nombre, se plantean las potencialidades de la filosofía "transescolar" en la formación docente. En él, a modo de ampliar la perspectiva de análisis, se toma en cuenta la noción de situacionalidad a través de los aportes de la didáctica -tanto general como específica- y de la filosofía latinoamericana. Así, el artículo se cierra con la clara postura de imbricar una discusión conceptual con los planos prácticos y experienciales fundados tanto en la tarea docente como de extensión universitaria de las autoras.

\section{Las prácticas filosóficas institucionales alternativas desde la filosofía filosofante}

El problema de la filosofía como objeto de enseñanza y aprendizaje ha girado canónicamente en torno a la polémica centrada en las posturas de enseñar filosofía versus enseñar a filosofar. Esta puede ser entendida como una antinomia o una falsa dicotomía, pero no cabe duda del lugar tradicional que ocupan las posturas 
encarnadas por Hegel y Kant. El primero concibe la enseñanza de la filosofía centrada en la historia de la disciplina, apoyado en la tesis de que la conciencia tiene un despliegue histórico y que en el desarrollo de la historia de la filosofía se encuentra la historia de la razón. Por su parte, Kant, al considerar que la historia no configura un conocimiento, sino un uso procedimental de la razón, plantea la enseñanza del uso de la razón que orienta, al distanciarse de los hechos e instalar las preguntas del por qué y del para qué del conocimiento de los objetos.

En el tránsito de este debate, sin pretensiones de negar las tensiones, este artículo presenta una perspectiva de la enseñanza filosofante que se conceptualiza como transescolar. Esta perspectiva tiene por objeto dar cuenta de algunos campos novedosos de la filosofía y el filosofar y otros no tan nuevos, pero que comparten sendas poco transitadas por la disciplina y su actividad o que lo hacen de un modo distinto al de la tradición institucionalizada.

Un dato innegable del campo filosófico es que en las últimas décadas han surgido prácticas que exceden los muros de las aulas de las escuelas secundarias y del nivel superior. En la lista se pueden incluir, entre otros, cafés filosóficos, talleres de filosofía, filosofía en las cárceles, filosofía clínica o philosophical counseling, divulgación filosófica a nivel editorial y en los medios masivos de comunicación, el programa de Filosofía para Niños y la propuesta de filosofía con niños y niñas.

Para ilustrar lo afirmado, en Argentina se pueden rastrear estas prácticas alternativas en la presentación de experiencias de la labor filosófica dentro y fuera de las instituciones educativas tradicionales. Para ello se toman las Jornadas sobre la Enseñanza de la Filosofía que organiza la Facultad de Filosofía y Letras de la Universidad de Buenos Aires en sus últimas tres ediciones (2009, 2011 y 2014). Allí se encuentran experiencias muy variadas; por ejemplo: talleres de danza y filosofía, filosofía en espacios exteriores (filosofar en las calles) para profesionales, talleres extracurriculares de lógica y argumentación, pensar sobre y con el cuerpo, filosofía en el arte y arte en la filosofía, filosofar en entornos virtuales, filokinesis, filosofía y arquitectura, filosofía como viaje interior, talleres de escritura filosófica colectiva, filosofía en bicicleta, música y filosofía, entre otras. 
Estos modos de hacer filosofía han impactado no solo en la forma de concebir la enseñanza de la filosofía, sino a la propia filosofía como disciplina. Ello se revela en su tematización, mientras que su novedad se muestra en la dificultad de denominarlas, cuestión que va estrechamente ligada a su conceptualización. Por eso es atinente preguntarse si es lícito definir a todas aquellas prácticas desde la negación de lo instituido y cómo se pueden pensar y nombrar con una entidad propia, sin limitarse a recurrir a la ausencia de lo otro.

En efecto, no le hace mérito al lugar que se han hecho denominarlas desde la exterioridad o la negación en relación a prácticas de enseñanza sólidamente establecidas, como es el caso de las que tradicionalmente se desarrollan en la escuela y la academia. Por eso es menester superar la consideración de que la enseñanza de la filosofía y las prácticas que la sostienen pueden ser pensadas como parte de la filosofía académica o, en contraposición, de un polo denominado "filosofía extra-académica" (Ceppas, 2005) o "no académica" (Santiago, 2006). Ello ya ha sido visto por los autores de dichas nociones que, al formularlas, muestran lo incompleto de sus conceptualizaciones.

Gustavo Santiago (2006), en la búsqueda de un elemento canónico de la filosofía, examina distintos tópicos para encontrar una característica que la distinga. Ello pasa por las definiciones, el quehacer concreto de los y las filósofas, estos como sujetos de la filosofía, los textos y, finalmente, los y las lectoras. Sin embargo, esta búsqueda le resulta infructuosa, puesto que no hay una filosofia, ya que esta solo puede darse en plural. En esta pluralidad, Santiago considera que hay distintas formas de legitimación de las prácticas filosóficas que exceden a la cátedra universitaria.

Parajustificar su postura, da cuenta de lo que piensa como dos modelos rivales desde los inicios de la filosofía occidental y que denomina como modalidades "académica" y "no académica"; lo hace de la mano de dos de sus representantes paradigmáticos, Platón y Sócrates, a los que considera, más que personajes históricos, "personajes conceptuales". Estos encarnan, en la concepción historiográfica dominante, lo que Hadot (2006) denomina filosofía como discurso y filosofía como modo de vida. Sin embargo, cabe aclarar que para Hadot, cuya postura aqui se sigue, Platón y sus sucesores -los académicos- todavía subordinan el discurso filosófico a un modo de 
vida, que es el objetivo final de toda la filosofía clásica y tardo antigua englobado en el eudemonismo.

A modo de cierre de su exposición, Santiago afirma:

Con el correr del tiempo, la concepción académica fue ganando terreno en relación con la «no académica» hasta que, fundamentalmente desde la modernidad, pasó a convertirse prácticamente en un sinónimo de la filosofía misma. El hecho de que no contemos con un nombre apropiado para referirnos de un modo "positivo" a su modalidad rival, teniendo que conformarse con "no académica", es un claro indicio de esa situación (2006, p. 27).

Por su parte, Filipe Ceppas conviene que esta tensión no será fácil de zanjar si se buscan pensar filosóficamente prácticascatalogadas como diversas y "heterodoxas", en relación con lo que se hace en la universidad e incluso la enseñanza de la filosofía en el nivel secundario. De ahí que sostenga como razonable aceptar una primera definición de la práctica filosófica extra-académica como aquella que se da fuera de la universidad y que involucra, fundamentalmente, a no-universitarios, es decir, a todas aquellas personas que estén motivadas para conocer las reflexiones elaboradas por otros, para reflexionar sobre ellas y, esencialmente, para compartir sus propias reflexiones en público, independientemente de su formación escolar, su ocupación o ingreso ${ }^{4}$ (2005, p. 50). Sin embargo, señala que este tipo de prácticas no son necesariamente no-académicas, dado que mantienen relaciones de distinto tipo con la academia. Por eso realiza este cuestionamiento: "¿por qué no decirque una filosofía extra-académica no es ni nunca será algo diferente de aquello a lo que podriamos denominar, tout court, filosofía a secas?" (Ceppas, 2006, p. 51).

En el caso de las autoras de este trabajo, la búsqueda de un término que pueda contener prácticas alternativas es producto de la actividad teórico-práctica en ámbitos institucionales diversos y de distinto grado de formalización educativa, conjuntamente con otras y otros que no están formados o (des)formados en el ámbito de la filosofía. Consecuentemente, la concepción del término transescolar no es una mera disquisición intelectual. Aqui vale pensar con El Poeta: "Si (como afirma

4 Las cursivas son de las autoras. 
el griego en el Cratilo) / el nombre es arquetipo de la cosa/ en las letras de 'rosa' está la rosa/ y todo el Nilo en la palabra 'Nilo'" (Borges, 1974, p. 885).

Con el fin de dar el contexto de producción de la idea de transescolaridad, se considera relevante mostrar cómo se fue delineando la noción de filosofía filosofante, surgida de un grupo de extensión universitaria denominado "Filosofía con niños, niñas y adolescentes. Hacia nuevas prácticas de articulación del espacio público educativo". Al momento fundacional de plasmar en el papel una enseñanza filosofante, se sostuvo como objetivo resignificar el concepto de filosofía desde el marco de la filosofía filosofante en contextos institucionales de distintos niveles educativos (Agudo et al., 2006). A diferencia de otras visiones que conciben una teoría y después la aplican, esta construcción surge como una noción deseable, a partir de un problema práctico: una insatisfacción como docentes y alumnas, un problema sobre la enseñanza de la filosofía. El problema no fue elucubrar una nueva noción de filosofía, sino poner en juego otra forma de hacerla. Pero esto no estaba reducido a una faz técnica, ya que hay una búsqueda de sentido, tal como lo explicita la idea de "resignificación" que aparece enunciada en el objetivo.

A la vez, si bien hay un esbozo teórico cuando en ese mismo objetivo se habla de la noción de filosofía filosofante, mediaron cinco años desde su enunciación a su delimitación conceptual. Ello porque ha sido fruto de una construcción dada por experiencias teóricas y prácticas en el encuentro con otros y otras; una experiencia intersubjetiva que apela, por un lado y en el plano técnico, a la intervención y, por otro lado y en el plano de lo público y lo individual, a la transformación de sentido. Este concepto se ha tejido, asi, en redes de prácticas y experiencias, pues uno de los objetivos de trabajo del mencionado grupo es propiciar redes interinstitucionales (De la Fuente y Morales, 2015).

Finalmente, es de destacar cómo se precisó la noción en el trabajo de escritura en la investigación; la escritura -como técnica- ha quedado en manos de algunas integrantes del grupo, por cuestiones que atañen a la división del trabajo desde una perspectiva de la cultura de la tarea (Elliot, 1997), aunque la construcción teórica es producto de un hacer colectivo. 
Si hasta aqui se ha hecho un tratamiento de la filosofía filosofante, en tanto marco de creación de la noción de transescolaridad, es porque esta es su fuente: se opone al academicismo que momifica las prácticas del pensar, por centrarse exclusivamente en el objeto y negar al sujeto que lo hace al olvidarse de él y de su actividad (Morales y Rodríguez, 2011). Pero como las instituciones son estructuras a las que de algún modo hay que adaptarse, si se pretende un tipo de inserción creativa en ellas, la filosofía filosofante no está en contra de la presencia de la filosofía en el espacio público académico. La academia, en tanto espacio público, permite, sin negar tensiones, abrir una fisura para la deslegitimación de prácticas academicistas y escolarizantes, ya criticadas, en términos kantianos, a través del concepto escolar de filosofía. Este se opone al concepto mundano como ciencia de los últimos fines de la razón humana. Este elevado concepto da a la filosofía dignidad, esto es, un valor absoluto. $Y$ efectivamente es también ella sola quien tiene únicamente valor interno, y sólo ella da valor a todos los demás conocimientos.

Sin embargo, al final, siempre ronda esta pregunta: ¿para qué sirve el filosofar y el fin último del mismo, la filosofía misma como ciencia, considerada según el concepto escolar? (Kant, 1943, pp. 42-43). A la respuesta que tiene una significación escolástica y que reduce la filosofía a mera utilidad, se le suma aqui otra respuesta, cuyo origen está en el interrogante de qué sucede si en vez de desestimar el concepto escolar se lo trans-forma.

\section{La perspectiva transescolar}

La investigación, la labor de enseñanza de la filosofía y el campo de su didáctica, habilitan al cuestionamiento de cómo nombrar aquello que tradicionalmente no es pensado como una práctica de enseñanza de la filosofía en la escuela o la academia. En un primer momento, se pensó esta diferenciación desde el eje de la institucionalización o no de la enseñanza filosofante; sin embargo, ello peca de ingenuidad: pensar que es posible salirse de la institucionalidad o que el centro de su potencia es estar en sus bordes. En un segundo momento, de la mano de la conceptualización de Sirvent et alt (2006), se amplió la mirada pensando la enseñanza filosofante desde distintos grados de formalización, en lugar de la polarización formal y no formal. 
La reflexión en torno a ello mostró la necesidad de generar una nueva conceptualización propositiva: una forma de entender la enseñanza filosofante desde una perspectiva que licue el binomio académica-no académica y, a su vez, que afirme a la filosofía filosofante en oposición a una perspectiva academicista y escolarizante de la disciplina. De alli la apuesta por la perspectiva transescolar.

Es Transescolar, pues al hablar de escuela se da cabida a la carga semántica de este término desde sus múltiples acepciones. En este sentido, se busca ir más allá de la escuela como es entendida habitualmente, en referencia al modelo institucional surgido en la modernidad. Asi, el calificativo transescolar marca lo que comparten todas las prácticas filosofantes a partir del término "escuela" y su devenir histórico. "Escuela" proviene del término latino schola y este del griego $\sigma \chi 0 \lambda \eta$, cuyo significado era el de tiempo libre para especular. El impacto de este vocablo se ve en la riqueza de sus acepciones en la lengua española y permite dar cuenta del complejo entramado de prácticas y tradiciones al que se busca remitir y que se señala como perspectiva transescolar.

Por ello, se establecerán relaciones a partir de algunas de las acepciones de la palabra "escuela" mencionadas en el diccionario de la Real Academia Española (RAE) -en su versión de 2014- con diversos conceptos que permitirán enriquecer y dar consistencia a la idea de transescolaridad. Si bien el concepto cuenta con cuenta con diez acepciones, en virtud del objetivo de este artículo, se tomarán solo algunas de ellas, aunque manteniendo la numeración original del diccionario de la RAE.

Las dos primeras significaciones establecen lo siguiente: 1) "Establecimiento público donde se da a los niños la instrucción primaria". 2) "Establecimiento o institución donde se dan o se reciben ciertos tipos de instrucción". La primera acepción, no casualmente, ocupa ese lugar, pues refiere al sentido de la educación institucionalizada a partir de la modernidad; es decir, la que aún subsiste, en líneas generales, en nuestra contemporaneidad. En cuanto a su condición de pública, señala el aspecto político de la institución: los lazos comunes y societales que exceden a los privados/particulares. Si se vincula la segunda acepción con la primera, aparece en ambas la idea de instrucción entendida como recorte de disciplinas, contenidos y prácticas de enseñanza y aprendizajes; estos, al ser considerados valiosos o socialmente relevantes, son regulados, en distinto grado, por el Estado. 
Para dar cuenta de esta condición, conviene aquí valerse de una palabra que está cargada de sentido, pero que no dice nada en la actualidad: res publica. El tratamiento paradigmático que ha tenido la instrucción pública en Condorcet servirá de piedra de toque para la comprensión de dicha palabra. El filósofo ilustrado, en sus Cincos memorias sobre la instrucción pública (2008), escritas en 1791, planteó ideas que sirvieron de base para el proyecto de organización del sistema educativo francés, presentado a la Asamblea Nacional en 1792, en el acmé de la Revolución Francesa.

La primera memoria, "Naturaleza y objeto de la instrucción pública", establece que es tarea y deber del Estado asegurar la instrucción del pueblo. Ello en virtud de que no es una cuestión de orden organizativo, sino una tarea política que permitirá hacer efectivos los derechos consagrados por la Revolución. Condorcet considera que la instrucción pública permitirá la igualdad, ya que la ignorancia y la desigualdad en la instrucción son algunas de las principales causas de la tiranía; es decir, la negación por antonomasia de un común. En síntesis, el Estado tiene que garantizar la instrucción, porque esta es la que garantizará la república.

En las obras de algunos de los clásicos latinos, la noción de res publica se traduce habitualmente como Estado o estado. En el primer caso, el más habitual, se hace presente la idea del Estado liberal, burgués, capitalista; en el segundo, entre tanto, hay un grado extremo de vaguedad, por lo que ambos no dan cuenta del sentido del término para los clásicos. Por ejemplo, Séneca plantea que existen dos tipos de res publica: la res publica particular, situada en los límites de aquellos lazos más estrechos que nos hacen ciudadanos y en virtud de los que, como individuo, se contempla y actúa en la particularidad; y otra, la res publica en relación con la totalidad, en la que el individuo contempla y hace en el cosmos (Séneca, 1932, p. 188). Estas dos formas de la res publica están intimamente asociadas, cuestión que para el imaginario social y la experiencia moderna y de nuestra contemporaneidad no es posible mentar.

Esta noción senequiana de res publica habilita para dar cuenta de intervenciones en torno a la producción de sentidos desde la filosofía y su enseñanza, no solo desde lo público y lo privado, sino desde las relaciones de experticia en torno a ellas en los distintos espacios en que se practican. Se concibe aqui a la res publica como 
dominio y construcción de lo común y no como Estado opuesto a la sociedad civil, tal como la comprendió la modernidad y que devino en los totalitarismos políticoeconómicos que se denuncian incesantemente desde las filosofías críticas; sin embargo, es menester aclarar que estas engloban una tradición mucho más extensa que la Escuela de Frankfurt e incluye las perspectivas que se afilian a una ontología del presente (Foucault, 1999).

Otra acepción de "escuela" es la de: 3) "Enseñanza que se da o que se adquiere". Este significado resulta lo suficientemente amplio como para incluir otro tipo de prácticas e instituciones distintas a la escuela moderna. La educación es un fenómeno más abarcador que el que implica la enseñanza en instituciones construidas especificamente para tal fin. Sin lugar a dudas, existe una diferencia relevante entre aquellas actividades que intencionalmente pretenden la enseñanza -y que realizan una secuenciación y organización gradual del proceso, con mayor o menor grado de formalización- y aquellas que acontecen espontáneamente. Esta acepción de "escuela" incluye ambos tipos de enseñanzas, así como otras que puedan mediar entre ambos polos extremos.

Por otro lado, hay una acepción vinculada a los modos didácticos de quien enseña: 5) "Método, estilo o gusto peculiar de cada maestro para enseñar"5. Aquí se hace referencia tanto a un plano subjetivo, que concierne a decisiones personales, como al impacto que tiene en los y las otras. En este sentido, escuela es el posicionamiento que sostiene cada docente acerca de su tarea y los saberes que enseña, entre otras cosas. En el caso de la filosofía, como se ampliará más adelante, implicará una manera de concebir a la propia disciplina.

Las dos siguientes acepciones se encuentran asociadas a las principales ideas de ciertos autores, corrientes de pensamiento o a quienes se adhieren a ellas: 6) "Doctrina, principios y sistema de un autor o conjunto de autores". 7) "Conjunto de discipulos y seguidores de una persona o de su doctrina, su arte, etc". Así, escuelas son tanto los sistemas de ideas como las personas que las sostienen, ya sea en calidad de autores o adscriptos. En el campo de la filosofía, es muy frecuente el uso

5 Tal como se señal más arriba, solo se toman algunas de las acepciones de la palabra "escuela"; por ello la cuarta acepción no es tenida en cuenta, pues no responde al objetivo de delimitar a la filosofía desde la idea de transescolaridad. 
de ambos sentidos. Estos dos sentidos dan cuenta de la educación como factor de creación, recreación y reproducción de la cultura. El problema de la reproducción es central a partir de la crítica moderna y atañe a distintos aspectos y sentidos de la cultura.

Aquí, por el tratamiento de la idea de escuela como parte del concepto de transescolaridad, se pone el foco en la crítica a la escolástica, entendida tanto en el sentido estrecho de crítica a la philosophia perennis de los seguidores medievales de Aristóteles, como en sentido amplio, es decir, la denuncia de la esclerosis de teorias y prácticas y, por ende, su divorcio con la actualidad en que se repiten. En el último caso, esta actitud ha tenido sus exponentes más renombrados a nivel social en las vanguardias artísticas y políticas. Por otro lado, en oposición a ese conservadurismo, la idea de escuela en esta acepción da cuenta de un momento de creación, en tanto se inaugura una nueva forma de hacer, pensary/o ser en el mundo.

En este punto, se puede tomar, a modo de ejemplo, a la filosofía como campo de la cultura y la lectura que hace de ella Alan Badiou. Este retoma a uno de sus maestros, Louis Althusser, y a su consideración de que "la filosofía es siempre la misma cosa. En este caso, el problema del desarrollo de la filosofía es simple: el futuro de la filosofía es su pasado" (2007, p. 124). Es importante destacar que de este modo, al señalarse en una tradición, Badiou pone en juego las ideas que conllevan las acepciones aquí analizadas.

Para Althusser, la filosofía es pura repetición de lo mismo y, ante la pregunta de qué es lo mismo, Badiou considera que es un acto formalmente idéntico a lo largo de la historia de la filosofía. Un acto que consiste en reorganizar las experiencias tanto teoréticas como prácticas y, con ello, proponer una nueva división normativa dentro de las opiniones, para subvertir el orden intelectual dado y dar lugar a nuevos valores. La forma de hacerlo es dirigirse con libertad a todos, pero especialmente a la juventud: "pues un filósofo sabe perfectamente bien que los jóvenes tienen que tomar decisiones sobre sus vidas, y que ellos están generalmente mejor dispuestos a aceptar los riesgos de una revuelta lógica" (2007, p. 125). De esta forma, ya no serán sujetos adscriptos pasivamente a una propuesta, sino protagonistas de un riesgo. 
La siguiente acepción está muy relacionada con las anteriores, ya que da cuenta de la situacionalidad: 8) "En literatura y en arte, conjunto de rasgos comunes y distintivos que caracterizan las obras de un grupo, de una época o de una región". Este significado adquiere especial relevancia, dado que nos enfrenta a la pregunta de qué tienen en común todas estas prácticas transescolares. Este interrogante será parcialmente respondido en este trabajo, aunque cabe sostener la inquietud, pues en un campo que alberga prácticas tan disimiles resulta complejo poder construir una definición desde lo común.

Por último, para los fines de este artículo, se considera a la escuela como: 9) "Lugar real o ideal que puede modelary enriquecer la experiencia". La noción de experiencia resulta compleja y posee diversas interpretaciones en el campo de la filosofía y, aún más, si la pensamos en su relación con las prácticas y teorias del campo educativo, tales son los casos de John Dewey y Walter Kohan.

El pragmatismo realiza una crítica profunda a la filosofía, en tanto esta se presenta como un análisis escindido de la realidad. En tal sentido, propone un acercamiento de la disciplina con la vida práctica y, en particular, con la experiencia. A partir de tal línea de pensamiento, no resulta extraño que gran parte de la obra de Dewey (1967) esté dedicada a un análisis de la moral, la política y la educación y a la imbricación entre estos tres ejes.

La escuela debe hacer hincapié en la experiencia del niño, pues Dewey considera de fundamental importancia que aquella sea la encargada de ofrecer algo más que la transmisión de conocimiento y brindar instancias de experimentación que, orientadas por el método científico, permitan construir conocimiento. La experiencia del pensar será recorrida desde el descubrimiento de un problema, el planteamiento de una hipótesis como posible respuesta y su verificación mediante la acción.

En el caso de Kohan, la idea de experiencia se nutre del pensamiento foucaultiano (Foucault, 2003): una transformación tanto individual como colectiva que no conlleva instrucciones concretas o un método, sino una invitación, una apertura a lo desconocido. En este sentido, la experiencia es lo que nos incentiva a ir más allá de lo ya pensado y dejar-nos que las cosas nos pasen. Aqui el uso de la primera persona 
del plural no es un descuido estilístico, sino una marca de este posicionamiento teórico. De esta forma, la experiencia para Kohan se relaciona con la noción de infancia, entendida como novedad y no como una etapa cronológica de la vida. A su juicio, quien transita la infancia, "le]s aquel que piensa de nuevo y hace pensar de nuevo. Cada vez por primera vez. El mundo no es lo que pensamos. "Nuestra" historia está inacabada. La experiencia está abierta" (2004, p. 275).

Si bien ambas nociones de experiencia se encuentran alejadas de los requisitos de la escuela como educación institucionalizada, la postura de Dewey -en la medida en que reconoce la importancia de un método- es más cercana a la idea de escuela como modeladora de la experiencia, tal como la postula la novena acepción de la RAE. Por su parte, la postura de Kohan parece más cercana al sentido originario de $\sigma \chi 0 \lambda \eta \dot{n}$, en tanto tiempo libre necesario para llegar a ser libres. Sin embargo, para dar cuenta de aspectos no contemplados por este autor, vale la pena retomar la noción de res publica y sus dos dimensiones: el contemplar y el hacer (rerum contemplationi et actioni) (Séneca, 1932, p. 190).

La res publica conlleva un determinado discurso sobre la escuela que no se agota ni en la teoría ni en la intervención, ya que produce sentidos y por ello es una práctica de la cual se tiene o no una experiencia. Estas palabras de Arendt pueden reseñar lo dicho: "El pensamiento mismo nace de los acontecimientos de la experiencia viva y debe mantenerse vinculado a ellos como los únicos indicadores para poder orientarse" (1997, p. 20).

Pero ¿cómo construir, transmitir y compartir una experiencia en nuestra contemporaneidad radicalmente instrumentalizada y, especificamente, desde la filosofía y su enseñanza? Para ello, y a modo de pie para lo que se desarrollará luego, cabe dar cuenta de los planos de la experiencia. Uno se encuentra centrado en la dimensión subjetiva, emparentada con lo sublime kantiano; es de índole individual e intransferible y va en el camino del mejoramiento de uno mismo (López, 2008). El otro da cuenta de la experiencia centrada en la intersubjetividad y, al entender de quienes aqui escriben, ha sido descuidado en el debate filosófico-educativo de los últimos 30 años; fundamentalmente, en el campo de pensamiento que considera dar lugar a la irrupción de lo nuevo en detrimento de las fijezas conceptuales. 
Este plano es el de la experiencia de la vida militante: demanda intervenir en el mundo, se vuelca a la exterioridad, al mundo como totalidad, como completitud y busca su mejoramiento y, por ello, en tanto se da una batalla para lograrlo, cabe asociarlo con la raíz que da entre otros términos al de miles, milites: soldado (Morales, 2014).

Terminado el tratamiento de algunas acepciones del término "escuela", es necesario dar cuenta del uso del prefijo trans como modalizador que excede el sentido locativo en el concepto de transescolaridad. Si se remonta al comienzo histórico de la escuela, esta se origina a partir de un desplazamiento, un movimiento. La Grecia aristocrática dio su lugar, luego de distintos conflictos sociales, a la Atenas democrática. Esta, entre otras cuestiones, produjo un cambio significativo en el modo de enseñar a los niños que aprendian saberes de manera sistemática. Ya estos no recibian lecciones en sus casas particulares, pues aumentó la cantidad de alumnos y, por ello, se tuvieron que desplazar a la casa del maestro (Plato, Protagoras, 326d).

A su vez, este movimiento de traslado espacial se acompaña de otro interno, nodal para esta forma de educación y que demanda esfuerzo. La $\sigma \chi 0 \lambda \dot{\eta}$ es la libertad para ser libre, en oposición a las expresiones tormento (tripalium) -que en español da la palabra trabajo- y desliz (labor) (Castello y Mársico, 2005). Sin embargo, sea entendida como condición de posibilidad para los estudios liberales (Marrou, 1975) o como la educación tradicional griega del hombre libre (Hadot, 2005), el ejercicio de tránsito hacia y por la escuela es la educación para el logro de la libertad del sujeto activo. Por esta razón $\sigma \chi 0 \lambda \eta$ se relaciona con esfuerzo, tal como lo indica la etimología de studium (Marrou, 1975). No hay que confundir el tiempo libre-del que se dispone para algo distinto al logro de la subsistencia- con la falta de esfuerzo o celo: toda exercitatio -y, más aún, la de los ejercicios espirituales cuyo fin es la felicidad del sujeto activo (Hadot, 2006)- demanda un empeño sostenido en el tiempo cronológico. Este ocio creador, posible en el tiempo de la experiencia, conlleva el esfuerzo de la transformación como movimiento intrasubjetivo.

Esta forma de concebir la escuela como movimiento permite aunar el concepto con el prefijo trans, en tanto que este no solo significa estar más allá, sino dar cuenta de ese movimiento que atraviesa todas las prácticas transescolares. De allí que la 
filosofía desde una perspectiva transescolar abarca dos aspectos: uno es de carácter intrasubjetivo y otro intersubjetivo.

El primero tiene que ver con quien realiza este desplazamiento. Para que ello ocurra, cada docente se hará cuestionamientos en relación con lo que pretende enseñar $y$, en función de esto, tomará decisiones filosóficas. Según Alejandro Cerletti, quien pretende enseñar filosofia, previamente deberá interrogarse qué es para él o ella la filosofía, cuál es su propia concepción, ya que de ello dependerá la modalidad que adopte para su enseñanza. "En cualquier situación de enseñanza de filosofía, lo que emerge siempre, se quiera hacer evidente o no, es la concepción de filosofía de quien asume la función de enseñar" (Cerletti, 2009, p. 83). A esta acepción de schola subyace el vínculo entre el compromiso filosófico y el pedagógico-didáctico intrasubjetivo, ya que los posicionamientos que se sostengan con el propio modo de entender la filosofía, traerán aparejadas decisiones pedagógicas que se traducirán en propuestas didácticas.

El otro aspecto es de carácter intersubjetivo y remite al sentido del prefijo trans como de transformación, esto es, como cambios relacionados con los lazos societales. La perspectiva transescolar puede pensarse como aquella que habilita prácticas filosóficas que permitirian transformar la escuela o que nos devolverian una mirada diferente de las instituciones educativas. La filosofía academicista es como una fuente cuyas aguas no se renuevan ni cambian, no reconoce contextos ni interlocutores, pues solo se interesa por si misma. La filosofía desde una perspectiva transescolar se distingue de ella, precisamente, por la práctica del filosofar que asimila contextos y dialoga con diversos interlocutores, sin ser la misma en cada uno de ellos. Aunque todas las prácticas transescolares se originan en contextos instituidos por el canon y se salen de este, algunas no están centradas en el aspecto crítico político y otras ni siquiera lo contemplan.

La noción de transescolaridad, como atributo de la filosofía, lleva a los partícipes de la educación institucionalizada al desafío de no invalidar o desmerecer prácticas que le son ajenas. Ello es posible si se tiene una mirada crítica de las instituciones, en tanto estas son reguladas también por marcos macropolíticos que establecen qué es la filosofía y cómo y quiénes la hacen. 


\section{Aportes y rupturas de la perspectiva transescolar a la formación}

La didáctica especíica de la filosofía se enfrenta con un gran desafío cuando, al momento de entrar en contacto con el campo de la enseñanza de la filosofia, el alumnado tiene una fuerte impronta academicista y una mirada de los fenómenos educativos encorsetada en su propia formación disciplinar. ¿Qué rupturas y posibles aportes propone la perspectiva transescolar en la formación en general y la docente en particular?

A lo largo de la formación docente, si es producto del tipo de currículum cerrado y tubular, se adquieren distintos enfoques, perspectivas y conocimientos de manera compartimentada, que pocas veces entran en diálogo, sino más bien todo lo contrario, ya que, al enaltecer su especificidad, pugnan por preponderar. Desde un espacio como la didáctica específica, se marca un quiebre con esta formación, en la medida en que ese bagaje heterogéneo de saberes necesariamente debe comenzar a modelarse, relacionarse y vincularse entre sí para dar lugar a una experiencia de formación.

En esa perspectiva de la formación, alumnos y alumnas recibendiversas respuestas, reflexiones, pensamientos en torno a la filosofía $y$, quizás, no se ha tenido tiempo y oportunidad de dedicarse a mantener y generar preguntas propias. Una perspectiva transescolar, en tanto asume la fuerza del sujeto como generador de prácticas, de pensamientos, no solo invita sino que exige un posicionamiento epistemológicorespecto de qué es la filosofía para cada uno, para luego considerar por qué y de qué modo enseñarla; es decir, pone en situación de edificar las propias decisiones a partir del acto mismo de filosofar.

Por lo contrario, desde el currículum de colección se piensa el perfil de los egresados y egresadas como ejecutoras que aplican los saberes de manera técnica, sin contextualizar ni vincular, ya sea en la tradición de enseñar filosofía como una galería de autores o sistemas (Sánchez Meca, 1996); o bien, como supuesta alternativa, un perfil docente que concibe enseñar a filosofar como adiestramiento en los procedimientos lógicos y argumentales. Por su parte, la perspectiva transescolar está atravesada y movilizada por el contexto y también lo atraviesa, de ahí que 
exige un posicionamiento político, un compromiso con lo común. Así resuenan las palabras de Kant (1943) en relación con el uso cósmico de la razón, aunque se diferencia de él en que esta posición no es hegemonizante ni docta, en la medida en que genera un espacio de diálogo con los distintos campos de la experiencia humana, al respetar la horizontalidad de miradas y voces que moldean y configuran los saberes.

Este atravesamiento del y por el contexto pone en juego una didáctica situada en un doble sentido: el de la filosofía latinoamericana y el de las ciencias de la educación. De la primera, la perspectiva transescolar toma la noción de pensamiento situado para pensar a la filosofía como un campo que aporta recursos para transformar nuestra situación de cultura subalterna (Morales y Rodríguez, 2011), mientras que de las ciencias de la educación se nutre de la idea de que la didáctica es producto de teorizar la enseñanza, que si bien es genérica, siempre se da en una situación institucional y organizacional particular (Mastache, 2014).

En sintesis, la perspectiva transescolar enfrenta las prácticas institucionales que al momento del ingreso a la docencia producen un wash out de la formación de grado (Zeichner en Davini, 2002). Esta idea es muy potente como metáfora de la incidencia de la biografía escolar previa y del desempeño del rol. Sin embargo, las instituciones también son moldeadas por los distintos sujetos y, en este sentido, asumir una mirada transescolar expone la posibilidad de serlo de manera activa al explorar y (re) conocer qué formación(es) se ha(n) tenido y qué se hará con ella(s) en la escuela; así, como también, de qué modo la formación favorece o limita la propia experiencia al ejercer la enseñanza filosofante, es decir, el oficio de ser profesores y profesoras filósofas.

Si desde el campo de la formación docente se amplía la interrogación a otros campos, cabe responder: hay que liberarse del canon como atadura, lo que permite generar nuevos contactos, tejer nuevas redes donde otros saberes, tanto disciplinares como sociales, permiten nuevas posibilidades de articular teoria, práctica y experiencia. 


\section{Referencias}

Agudo, S., et al. (2006). Proyecto de extensión universitaria Filosofia con niñas, niños y adolescentes: hacia la configuración de prácticas de articulación en el espacio público educativo. Bahía Blanca: Departamento de Humanidades-Universidad Nacional del Sur.

Arendt, H. (1997). Barcelona: Paidós.

Badiou, A. (2007). La filosofía como repetición creativa. Acontecimiento, XVII(33-34), pp. 123-131.

Borges, J. (1974). El Golem. Obras completas (pp. 885-887). Buenos Aires: Emecé.

Castello, L., y Mársico, C. (2005). Diccionario etimológico de términos usuales en la práctica docente. Buenos Aires: Altamira.

Ceppas, F. (2005). Sobre las prácticas extra-académicas. Novedades Educativas, 17(169), pp. 50-54.

Cerletti, A. (2009). Repetición, novedad y sujeto en la educación. Buenos Aires: Del Estante.

Condorcet, J. (2008) Cinco memórias sobre a instrução pública. (Trad. M. de Souza.). São Paulo: Editora Unesp.

Davini, M. C. (2002). De aprendices a maestros. Enseñar y aprender a enseñar. Buenos Aires: Papers Editores.

De la Fuente, L., y Morales, L. (2015). La experiencia de la lectura como forma de repensar las relaciones entre la investigación y la extensión universitaria. L. de la Fuente y L. Morales (Eds.), Volúmenes Temáticos de las $V$ Jornadas de Investigación en Humanidades. Vol. 2., (pp. 17-24). Bahía Blanca: Hemisferio Derecho.

Dewey, J. (1967). Democracia y educación. Buenos Aires: Losada.

Elliot, J. (1997). La investigación-acción en educación. (Trad. P. Manzano). Madrid: Morata.

Foucault, M. (1999). Estética, ética y hermenéutica. Barcelona: Paidós.

Foucault, M. (2003). Yo minimalista y otras conversaciones. Buenos Aires: La marca. 
Hadot, I. (2005). Arts libéraux et philosophie dans la pensée antique. Contribution da l'histoire de l'éducation et de la culture dans l'Antiquité. Paris: Vrin.

Hadot, P. (2006). Ejercicios espirituales. (Trad. J. Palacio). Madrid: Ediciones Siruela. Kant, I. (1943). Sobre el saber filosófico. (Trad. J. Marias). Madrid: Adán.

Kohan, Walter. (2004). Infancia. Entre educación y filosofía. Barcelona: Laertes.

López, M. (2008). Filosofía con niños y jóvenes. La comunidad de indagación a partir de los conceptos de acontecimiento y experiencia trágica. Buenos Aires: Novedades Educativas.

Marrou, H. (1975). Historia de la educación en la antigüedad. (Trad. J. Mayo). Buenos Aires: Eudeba.

Mastache, A. (2014). La didáctica de nivel medio como sintesis de saberes. Imágenes de la Didáctica de Nivel Medio. A. M. Malet., y E. Monetti (Eds.), Debates universitarios acerca de lo didáctico y la formación docente (pp. 81-87). Buenos Aires: Noveduc.

Morales, L., y Rodríguez, L. (2011). La filosofia en los contextos de las politicas curriculares desde los 90. Un análisis desde la filosofia filosofante. Ponencia presentada en XVII Jornadas sobre enseñanza de la Filosofía. Buenos Aires.

Morales, L. (2013). Prácticas filosóficas y trabajo en red. Revista de la Red Institucional de Filosofia con niños y adolescentes, (2), pp. 53-56.

Plato. (1976). Protagoras. (Trad. C. Taylor). Oxford: Clarendon Press.

Real Academia Española. (2014). Diccionario de la lengua española. Revisado el 30 de julio de 2018 en $h$ ttp://dle.rae.es/?id=GMFMuVV

Sánchez Meca, D. (1996). La historia de la filosofía como hermenéutica. Madrid: Universidad Nacional de Educación a Distancia.

Santiago, G. (2006). Filosofía, niños, escuela. Trabajar por un encuentro intenso. Buenos Aires: Paidós.

Seneca (1932). Moral Essays: volume 2 (pp.182-200. London and New York: Heinemann. 1932.

Sirvent, M. T., et al. (2006). Revisión del concepto de educación no formal. Cuadernos de Cátedra de Educación No Formal (pp. 3-21). Buenos Aires: Facultad de Filosofía y Letras UBA. 